\title{
ARTICLE OPEN Revealing region-specific biofilm viscoelastic properties by means of a micro-rheological approach
}

\author{
Huayu Cao ${ }^{1}$, Olivier Habimana ${ }^{2}$, Ashkan Safari ${ }^{1}$, Rory Heffernan ${ }^{1}$, Yihong Dai ${ }^{1}$ and Eoin Casey ${ }^{1}$
}

Particle-tracking microrheology is an in situ technique that allows quantification of biofilm material properties. It overcomes the limitations of alternative techniques such as bulk rheology or force spectroscopy by providing data on region specific material properties at any required biofilm location and can be combined with confocal microscopy and associated structural analysis. This article describes single particle tracking microrheology combined with confocal laser scanning microscopy to resolve the biofilm structure in 3 dimensions and calculate the creep compliances locally. Samples were analysed from Pseudomonas fluorescens biofilms that were cultivated over two timescales $(24 \mathrm{~h}$ and $48 \mathrm{~h}$ ) and alternate ionic conditions (with and without calcium chloride supplementation). The region-based creep compliance analysis showed that the creep compliance of biofilm void zones is the primary contributor to biofilm mechanical properties, contributing to the overall viscoelastic character.

npj Biofilms and Microbiomes (2016)2:5; doi:10.1038/s41522-016-0005-y

\section{INTRODUCTION}

The characterisation of biofilms in terms of their material properties is important for the fundamental understanding of methods for the control of unwanted biofilms.1, 2 However comprehensive quantification of the mechanical properties of biofilm remains a major challenge, in part due to the temporal, spatial and compositional dynamics of the extracellular matrix, ${ }^{3}$ but also due to the lack of available techniques for routine laboratory measurements. Some methods, such as atomic force microscopy $(\mathrm{AFM})^{4}$ or the parallel plate rheometer ${ }^{5}$ have been successfully applied for investigation of the structural and physical properties, however they are limited in their ability to describe properties at acceptable resolution. For example, AFM-based nano-indentation of mature biofilms is limited to the examination of the biofilm-liquid interface region of a sample, ${ }^{4}$ whereas standard rheometer measurements describe the entire biofilm sample but provide only bulk average properties and, in most cases, require ex situ analysis and a quantity of biofilm sample that may not be practical.

Particle-tracking micro-rheology, which resolves the local mechanical properties spatially and temporally, has emerged as a promising technique to depict the biofilm at microscale. ${ }^{6-10}$ Birjiniuk et al. found that charge interactions play a key role in mobility within biofilms. ${ }^{6}$ Chew et al. discovered how genetic changes influence the biofilm mechanical property and structure formation. In $P$. aeruginosa biofilms, the role of known polysaccharides was elucidated: Psl increased the elasticity and effective cross-linking within the matrix while Pel reduced effective cross-linking within the matrix. ${ }^{7}$ Another study used magnetic tweezers via carboxylated magnetic bead probes embedded in biofilm. It was found that the creep compliance become higher with increasing height from the bottom of biofilm. 8
Although each of these studies has provided insight into biofilm structure, there are few papers that quantitatively describe, for the same biofilm samples, both structural and mechanical properties in situ. This paper describes single particle tracking microrheology combined with confocal laser scanning microscopy to resolve the biofilm structure in three dimensions and calculate the creep compliances locally. Moreover, the biofilm was examined at different time scales ( $24 \mathrm{~h}$ and $48 \mathrm{~h}$ cultivation) and alternate environment conditions (with and without calcium chloride supplementation). The biofilm was analysed by classification into three layers (bottom, middle, top) depending on the normalized height and the tracking particles were segregated into two different biofilm regional characteristics (voids and clusters). This paper describes the heterogeneity of the biofilm structure and explains provides some data on the differences in material characteristics depending on cultivation time.

\section{MATERIALS AND METHODS}

\section{Bacterial strains}

The selected bacterial strain for this study was an mCherry-expressing $P$. fluorescens PCL1701, ${ }^{11}$ stored at $-80^{\circ} \mathrm{C}$ in King B broth ${ }^{12}$ supplemented with $20 \%$ glycerol. Cultures were obtained by inoculating $100 \mathrm{ml}$ of King B broth supplemented with gentamicin at a final concentration of $10 \mu \mathrm{g} \mathrm{ml}^{-1}$ using a single colony of a previously grown culture on King B agar (Sigma Aldrich, Ireland) at $28^{\circ} \mathrm{C}$. The inoculated medium was then incubated at $28^{\circ} \mathrm{C}$ with shaking at $75 \mathrm{rpm}$ and left to grow to late exponential growth phase, corresponding to an optical density $\left(\mathrm{OD}_{600}\right)$ of about 1.0.

\section{Fluorescent bead preparation}

Green fluorescent carboxylate micro beads (Sigma, L46) of $1 \mu \mathrm{m}$ diameter were used in all biofilm microrheological experiments. A concentrated micro bead solution was first diluted $(1: 30)$ in Grade 1 quality water $\left(18.2 \mathrm{M} \Omega \mathrm{cm}^{-1}\right)$ obtained from an Elga Process Water System (Biopure 15

\footnotetext{
${ }^{1}$ School of Chemical and Bioprocess Engineering, University College Dublin (UCD), Belfield, Dublin 4, Ireland and ${ }^{2}$ School of Biological Sciences, The University of Hong Kong, Hong Kong, PR China.

Correspondence: Eoin Casey (eoin.casey@ucd.ie)

Huayu Cao and Olivier Habimana contributed equally to this work
}

Received: 15 May 2016 Revised: 28 July 2016 Accepted: 5 August 2016

Published online: 05 December 2016 
and Pureflex 2, Veolia, Ireland), and will henceforth be referred to as MilliQ water. The suspension was then centrifuged at 10,000 RPM for $10 \mathrm{~min}$. The supernatant was carefully discarded and the micro bead re-suspended with $25 \mu \mathrm{l}$ volume of MilliQ. This sequence was repeated three times to remove any trace of surfactants from the solution the micro beads were provided in. Prior to biofilm growth experiments, micro bead pellets were re-suspended in sterile PBS-buffer solution.

\section{Biofilm growth and bead implantation}

A $4 \mu \mathrm{l}$ volume of an overnight culture of $P$. fluorescens was used to inoculate sterile individual centrifuge tubes (Falcon, Fischer Scientific, Ireland). Single autoclaved cover slips (Thermo Scientific, Germany, borosilicate glass $18 \times 24 \mathrm{~mm}$ ), were partially submerged into individual tubes. Biofilm that formed on these coverslips were the basis for all measurements in this study. Each tube contained an initial $3 \mathrm{ml}$ of King B broth supplemented with gentamicin at a final concentration of $10 \mu \mathrm{g} \mathrm{ml}^{-1}$. Previously prepared green fluorescent polystyrene $1 \mu \mathrm{m}$ diameter beads (Sigma, L46) were added into the King B medium to a final concentration of $5 \times 10^{5}$ beads $\mathrm{ml}^{-1}$. To assess biofilm under an alternative ionic environment, tubes were supplemented with $\mathrm{CaCl}_{2}$ at a final concentration of $15 \mathrm{mM}$. Control tubes were not supplemented with $\mathrm{CaCl}_{2}$. Experiments were performed in triplicate with independently grown $P$. fluorescens culture incubated for $24 \mathrm{~h}$ or $48 \mathrm{~h}$ at $28^{\circ} \mathrm{C}$ with shaking at $75 \mathrm{rpm}$.

\section{Confocal laser scanning microscopy}

Following biofilm growth, coverslips were first rinsed in sterile PBS solution by gently dipping the coverslip containing biofilm in a tube containing sterile $0.1 \mathrm{M} \mathrm{NaCl}$ solution. The coverslip was then carefully placed in a single-well Nunc Lab-Tek II Chamber Slide (VWR, Ireland) filled with sterile phosphate buffered saline solution.

Horizontal plane images (xy-plane) of the biofilms were acquired using an Olympus FV1000 CLSM at the Live Cell Imaging core technology facility at the UCD Conway Institute. CLSM experiments were repeated three times for each biofilm growth condition using independently grown $P$. fluorescens cultures. At least three to four random areas were acquired for each biofilm sample per experiment. The excitation wavelength used for mCherry expressing $P$. fluorescens cells was $559 \mathrm{~nm}$, and the emitted fluorescence was recorded within the range of $603 \mathrm{~nm}$. The green fluorescent beads within biofilms were excited at $488 \mathrm{~nm}$ and emissions registered at $519 \mathrm{~nm}$. Images were collected through an Olympus UPL SAPO $60 \times$ NA:1.35 Oil objective with a z-step of $1 \mu \mathrm{m}$. Additionally, for each selected random position, time series of $2.25 \mathrm{~s}$ scanning time-increments were separately acquired from bottom to top regions within the biofilm depth, termed as z-horizontal planes, for approximately $135 \mathrm{~s}$. Time series (xyt-) stacks were thereby composed of 60 slices and were analyzed to assess bead trajectory. 3D projections were performed using FIJI image processing tool's 3D viewer plugin.

\section{Biofilm structure analysis}

The structural quantification of biofilms was performed using the ISA3D MATLAB-based software developed by Beyenal et al., ${ }^{13}$ allowing a detailed analysis of biofilm structural and textural properties. The latter includes textual energy, a parameter introduced by Haralick et al. ${ }^{14}$ as a measure of directionally repeating patterns of pixels in images. Lower textual energy values indicate less frequent and fewer repeated patterns of pixel clusters in one direction, and higher textual energy shows a more homogeneous image structure with fewer repeated patterns. The term entropy was first defined in communication theory by Shannon in $1948,{ }^{15}$ and is a measure of uncertainty of information. Textural entropy in the context of biofilm samples as analysed by the ISA3D software represent the degree of randomness of pixels in the gray scale of image. Higher textual entropy values indicate increased levels of heterogeneity.

\section{Particle trajectory registration and Mean mean square}

displacement (MSD) analyses

Using the acquired CLSM xyt-time series stacks, particle trajectories were obtained using the Diatrack software (version 3.04 Pro). ${ }^{16}$ The obtained bead trajectories were used to calculate the MSDs as follows:

$\mathrm{MSD}=\left\langle\Delta r^{2}(\tau)\right\rangle=\langle r(t+\tau)-r(t)\rangle^{2}$

where $r$ represents the position of a particle at time $t$, and $\tau$ is the lag time. In this study, the MSDs describe how embedded beads in $P$. fluorescens biofilms move within the biomass. These recorded migrations are then used to compute localized viscoelastic properties of the biofilm material, in the form of creep compliance, by recording the MSDs over time. The MSDs were computationally determined using a Matlab script, developed for the purpose of this study (cf. Fig. S1. supplementary material section).

The creep compliance, which is defined as the ratio of displacement to a given applied force over time, can be calculated from the MSDs. The MSD is therefore proportional to the creep compliance of the material in which the beads were embedded, ${ }^{6}$ and is expressed by the following equation:

$J=\frac{3 \pi d}{4 k_{B} T}\left\langle\Delta r^{2}(t)\right\rangle$

where $J$ is the creep compliance, $d$ is the diameter of the polystyrene beads, $t$ is the temperature and $k_{B}$ is the Boltzmann constant.

This method offers a non-destructive method of measuring the material properties of biofilms at different horizontal z-planes (bottom, middle and top). In combination with data obtained with confocal microscopy, the structural and mechanical properties of biofilms may be simultaneously assessed to provide an insightful analysis of the spatiotemporal development of biofilms.

Developmental tool for the analysis of region-specific microrheology profiles

In order to classify beads into populations of confined and mobile populations based on obtained MSD data, an unbiased statistical model was used to group the two types of trajectories into two grouped populations. This is shown in supplementary Fig. S2 (cf. supplementary material section), where the product of the range $\left[\max \left(r_{x}\right)-\min \left(r_{x}\right)\right]$ and the standard deviation $\sigma_{\mathrm{x}}$ of the x-coordinate of the particle. To determine an approximate cut-off, the particles that fall above the dashed line are designated as mobile while the ones below the cut off line were designated as immobile or confined. ${ }^{17}$ Following creep compliance measurements of all beads within a given biofilm field of view, contour maps were created using MINITAB v15.1 (Minitab Inc., State College, PA), by applying Akima's polynomial method for the interpolation of creep compliance values for each fluorescent bead's $x$-y-coordinate.

\section{Statistical analysis}

One-way analysis of variance (ANOVA) was performed using MINITAB v15.1 (Minitab Inc., State College, PA) in order to test the significant differences in biofilm structural parameters of $P$. fluorescens biofilms grown in the presence or absence of $\mathrm{CaCl}_{2}$ with Tukey's test for pairwise comparisons. ANOVA was also performed to determine statistical differences in creep compliance $\left(\mathrm{Pa}^{-1}\right)$ of different biofilm layers and regions (voids and cell clusters) of $24 \mathrm{~h}$ - and $48 \mathrm{~h}$-grown $P$. fluorescens biofilms under the presence or absence of $\mathrm{CaCl}_{2}$. All tests were performed at the $5 \%$ significance level.

\section{RESULTS AND DISCUSSION}

\section{Biofilm structural and morphological properties}

The effect of $\mathrm{CaCl}_{2}$ on $P$. fluorescens biofilm structure and morphology was first assessed over a two day period, as previously described by Safari et al. ${ }^{4}$ However, a higher magnification during CLSM acquisitions was used to locate and monitor embedded fluorescent beads within the biofilms matrix. Fig. 1 describes representative three dimensional projections of $24 \mathrm{~h}$ and $48 \mathrm{~h}$ old Pseudomonas fluorescens biofilms obtained following growth at $0 \mathrm{mM} \mathrm{CaCl}$ or $15 \mathrm{mM} \mathrm{CaCl}$ environments. Biofilms generally appeared to have increased in surface coverage from $24 \mathrm{~h}$ and $48 \mathrm{~h}$ growth periods. Biofilms grown in the absence of $\mathrm{CaCl}_{2}$ appeared to be less confluent as biofilms grown in the presence of $\mathrm{CaCl}_{2}$, hence confirming results previously obtained in a similar study. ${ }^{4}$ Biofilm cells also appeared to cover more surface area in the presence of $\mathrm{CaCl}_{2}$. Structural differences were however not easily distinguishable between biofilms grown under different $\mathrm{CaCl}_{2}$ environments as previously described. ${ }^{4}$ This limitation could be explained by the $63 x$ lens used during this study, which allowed the acquisition of smaller biofilm areas with much higher magnification, as opposed to the $10 \times$ objective giving lower magnification and larger field of views. Although the qualitative analysis presented a number of limitations when comparing and interpreting acquired biofilms, the quantitative analyses of CLSM data using Image structure analysis tool allowed an in-depth study of the structural and textural biofilm properties in this study (Table $1 A B$ ). Structural biofilm properties such as biovolume and mean thickness increased, while decreasing in porosity and biofilm 
roughness parameters from $24 \mathrm{~h}$ to $48 \mathrm{~h}$ growth periods (Table $1 \mathrm{~A}$ ). However, these changes were insignificant $(p>0.05)$ in the absence of $\mathrm{CaCl}_{2}$. Changes in biofilm structure was found to be significant in the presence of $\mathrm{CaCl}_{2}$ from 24 to $48 \mathrm{~h}$ development for parameters such as biovolume $(p=0.003)$, mean thickness $(p=0.022)$, and biofilm roughness $(p=0.046)$, while the drop in biofilm porosity was insignificant $(p=0.29)$.

When comparing the effect of $\mathrm{CaCl}_{2}$ on both $24-\mathrm{h}$ and $48-\mathrm{h} P$. fluorescens biofilms, high calcium concentrations led to significant changes to structural and textural properties. The presence of $\mathrm{CaCl}_{2}$ contributed to increased biovolume $(p<0.05)$, lower biofilm porosity $(p<0.05)$ and roughness $(p<0.05)$. Mean thickness was shown to not have been significantly affected by the presence of $\mathrm{CaCl}_{2}$ in both $24 \mathrm{~h}$-and $48 \mathrm{~h}$ old biofilms with $p$ values of 0.293 and 0.523 , respectively.

Biofilm textural analysis (Table 1B) revealed that the presence of $\mathrm{CaCl}_{2}$ led to changes in the form of biofilm heterogeneity. This was particularly apparent by the increased textural entropy and lowered homogeneity parameters compared to growth in the absence of $\mathrm{CaCl}_{2}$. These results confirm the qualitative observations obtained in a previous study in which the presence of calcium led to an increase in global roughness. ${ }^{4}$ This heterogeneity was also determined by the energy parameter, ${ }^{13}$ which was found to be generally lower in the presence of $\mathrm{CaCl}_{2}$, indicating a higher frequency of repeating cluster patters, and therefore a more heterogeneous biofilm.
$24 \mathrm{~h}$
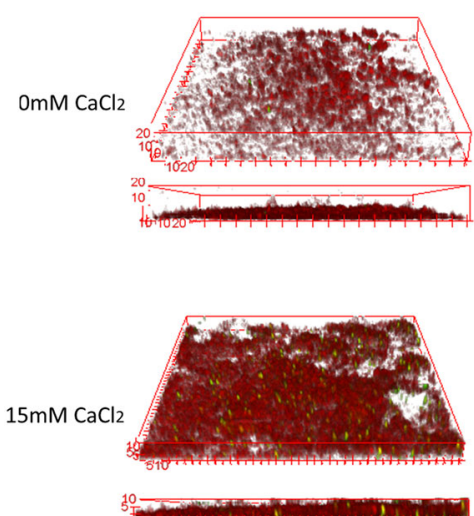

$48 \mathrm{~h}$
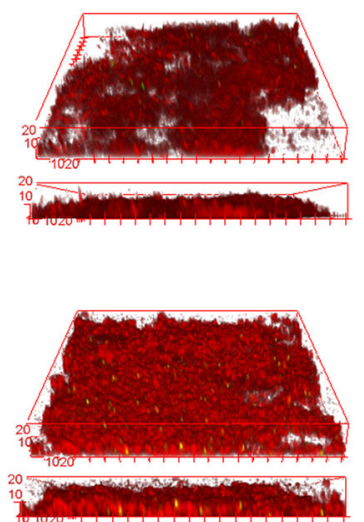

Fig. 1. Three dimensional (xyz) and corresponding two dimensional (xz) CLSM projections of $24 \mathrm{~h}$ and $48 \mathrm{~h}$ mCherry-expressing Pseudomonas fluorescens following growth in the absence $(0 \mathrm{mM})$ or presence $(15 \mathrm{mM})$ of $\mathrm{CaCl}_{2}$. The green dots are fluorescent polystyrene beads $(1 \mu \mathrm{m})$. 3D images were created with ImageJ's "3D viewer" plugin
Biofilms were grown in the presence of embedded fluorescent nanoparticles, with the objective of purposely analysing for subsequent microrheological studies. The advantage with this approach was the ability to measure beads throughout the biofilm depth, thereby providing a detailed viscoelastic characterization profile in a non-destructive in situ manner, as opposed to the traditional destructive classical rheology and force spectroscopy techniques. The beads in this study were uniformly embedded in the biofilms and their surface coverage throughout biofilm depth is presented in Fig. 2. The bead surface coverage parameter at various depths was found to be influenced by biofilm age $(p=0.018)$ and exposure to $\mathrm{CaCl}_{2}(p<0.001)$ during growth. In terms of bead localization, results showed that beads embedded in biofilm grown in the absence of $\mathrm{CaCl}_{2}$ were generally equally spread in the bottom and middle layers of the biofilm. The bead profile of biofilms grown in the presence of $\mathrm{CaCl}_{2}$ revealed a gradual decrease in bead surface coverage from bottom to top biofilm layers with the highest surface coverage at the bottom regions of the biofilms. These results suggest that the level of bead interaction during biofilm development is not only cultivation-time dependent but is also linked to the environment at the time of these interactions take place. The higher level of bead surface coverage within biofilms could have been attributed to the higher adhesive nature of $P$. fluorescens biofilms in the presence of $\mathrm{CaCl}_{2}$ as recently described, ${ }^{4}$ thereby promoting bead-biofilm interactions. The higher level of beads at the bottom layers also suggests that this interaction may have initially occurred at the onset of biofilm development. Interestingly, the decreasing bead surface coverage from bottom to top biofilm layers is indicative of possible variations in mechanical, and viscoelastic internal biofilm properties which can be better understood through microrheological techniques. A non-destructive in situ approach such described in this study is therefore useful in understanding potential mechanical and viscoelastic variation within the entire biofilm depth.

Micro-rheological analysis of biofilms

MSD. The mechanical properties of $24 \mathrm{~h}$ and $48 \mathrm{~h}$ biofilms formed in the presence or absence of $\mathrm{CaCl}_{2}$ were analysed by following the MSDs and creep compliance of $1 \mu \mathrm{m}$ polystyrene beads embedded different biofilm depths and regions. Biofilm layers were classified into bottom, middle and top zones according to normalized biofilm depths of $0-33,34-66$ and $67-100$, respectively. Vibrations from embedded $1.0 \mu \mathrm{m}$ beads at fixed positions were recorded and the analysed MSD values were typical of viscoelastic materials.

The MSDs of all tested biofilms (Figs. 3a, b, c, d) exhibited a viscoelastic profile based on the fitting of MSDsot ${ }^{a}$. The calculated exponent $a$ is superior to 0 (elastic) and 1 (viscous/liquid) (cf. Table S1. supplementary material section). Interestingly, MSD values indicated distinct rheological differences within biofilm layers over time depending on the salt environment in which the biofilms were grown.

In the absence of $\mathrm{CaCl}_{2}$ (Fig. 3a, b), the median MSDs ranged between $6.5 \times 10^{-3}$ to $8.26 \times 10^{-3} \mu^{2}$ within $24 \mathrm{~h}$ biofilms (Fig. 3a) to MSD values

\begin{tabular}{|llllllll}
\hline Table 1. Biofilm structural $(\mathrm{A})$ and textural (B) properties & & & & \\
\hline
\end{tabular}




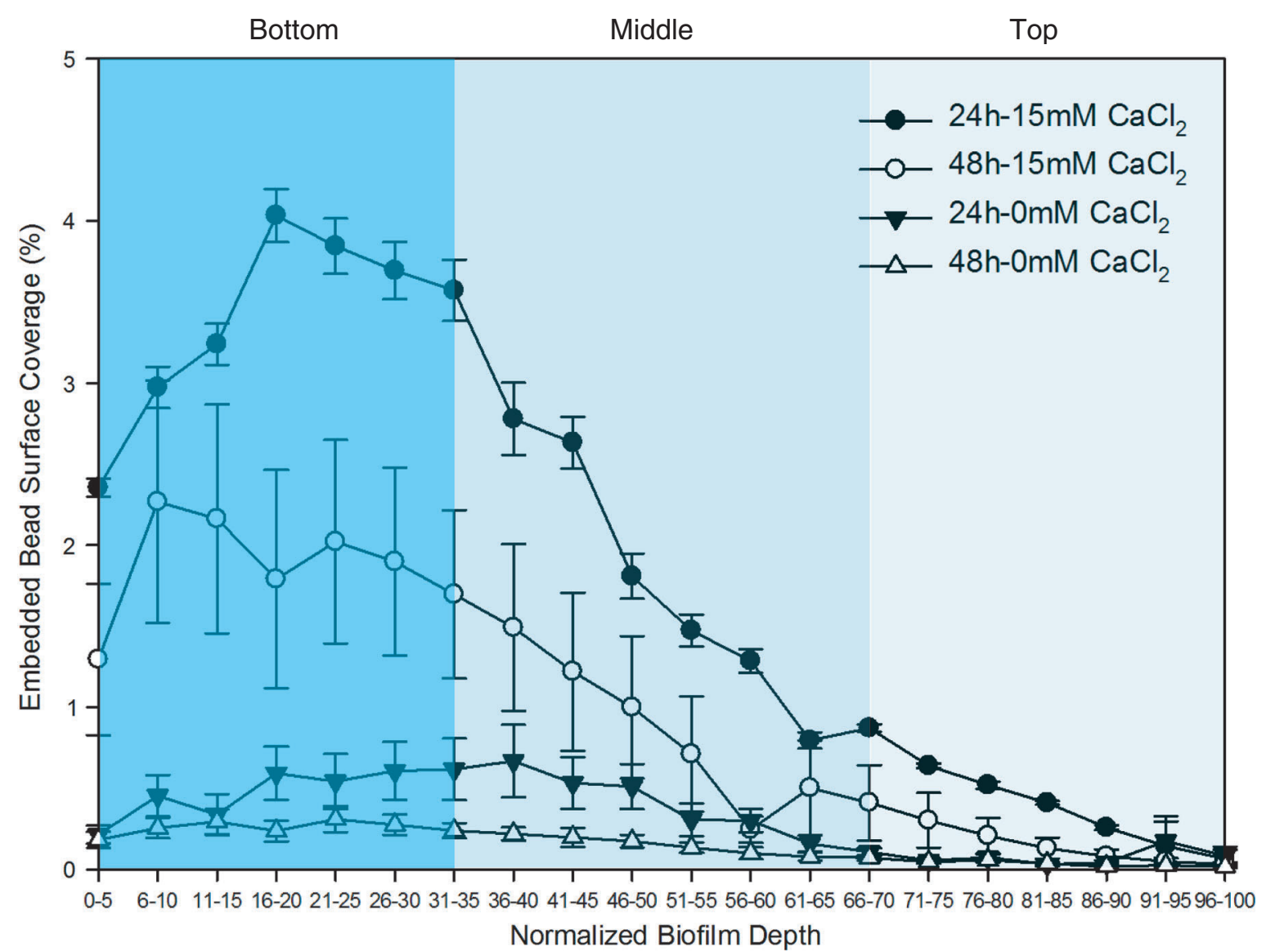

Fig. 2. Spatial stratification of embedded fluorescent $1 \mu \mathrm{m}$ beads presented as surface coverage (\%) vs. biofilm depth following $24 \mathrm{~h}$, $48 \mathrm{~h}$ biofilm development in the absence and presence of $\mathrm{CaCl}_{2}$. Error bars represent standard error of the mean

between $1.6 \times 10^{-2}$ and $1.2 \times 10^{-2} \mu \mathrm{m}^{2}$ within $48 \mathrm{~h}$ biofilms (Fig. $3 \mathrm{~b}$ ). This increase in MSD values within $48 \mathrm{~h}$ biofilms is an indicator of the increased ductile nature of the biofilm, through the loosening of biofilm matrix allowing greater bead displacement. Detailed MSD analysis of the different biofilm layers revealed that the top layer of $24 \mathrm{~h}$ biofilms grown in the absence of $\mathrm{CaCl}_{2}$ went from being the least malleable to the most malleable layer following $48 \mathrm{~h}$ growth. This change, observed by the increase MSD values, suggests that the rheological profile of the biofilm matrix is biofilm depth dependent, and may be linked to the changes in the chemical microenvironments associated with biofilm growth. ${ }^{18,} 19$

In the presence of $\mathrm{CaCl}_{2}$ (Fig. 3C, d), the median MSDs ranged between $1.6 \times 10^{-2}$ to $1.2 \times 10^{-2} \mu^{2}$ within $24 \mathrm{~h}$ biofilms (Fig. 3c) to MSD values between $6.9 \times 10^{-3}$ and $9.8 \times 10^{-3} \mu^{2}$ within $48 \mathrm{~h}$ biofilms (Fig. $3 \mathrm{~b}$ ). This decrease in MSDs between $24 \mathrm{~h}$ and $48 \mathrm{~h}$ growth periods is indicative of a generalised stiffening of the biofilm, through the increase in effective cross-linking in the matrix network within biofilms caused by the presence of calcium, known for its crosslinking and gel stiffening properties. Interestingly, the rheological properties of top biofilm layer went from being most malleable following $24 \mathrm{~h}$ to being the stiffest biofilm layer following $48 \mathrm{~h}$ development.

Comparing biofilms grown at different cultivation times, the presence of $\mathrm{CaCl}_{2}$ (Fig. 3c) generally led to more ductile biofilms following $24 \mathrm{~h}$ (Fig. 3a). Following longer growth periods, the presence of $\mathrm{CaCl}_{2}$ (Fig. 3d) led to a stiffer biofilm compared to growth in the absence of $\mathrm{CaCl}_{2}$ (Fig. 3b). These differences could be associated with the structure and maturity of biofilms as previously described. The stiffer biofilm in the absence of calcium following $24 \mathrm{~h}$ could be explained by the higher ratio of cells to EPS. ${ }^{4}$ Interestingly, after $48 \mathrm{~h}$, biofilms grown under calcium supplementation (Fig. 3d) were much stiffer than biofilm development without $\mathrm{CaCl}_{2}$ (Fig. 3c). Moreover, the rheological profile through the biofilm depth suggests that calcium led to stiffer top layers.

Creep Compliance. Creep compliance, which is a measurement of how materials deform over time, was calculated based on the MSD values for all biofilm samples to example the effect of cultivation time and $\mathrm{CaCl}_{2}$ concentration (Fig. 4). The analysis presented here allows quantification of the rheological properties in different layers within the same biofilm sample. Contrarily to what was observed with the MSD data, there were no statistical differences $(p>0.05)$ between bottom, middle and top layers for biofilms grown in their respective conditions. It should however be noted that the lack of creep compliance variations between the layers is separate to the heterogeneous and complex nature of the biofilm. The use of beads for the microrheological studies of biofilms should therefore not be limited to a layered biofilm classification, but rather, should selectively include regions within the biofilm. Since biofilms are structurally heterogeneous, MSDs and creep compliance data analysis should take this into consideration. A modified approach was therefore developed to analyse MSDS and creep compliance of key biofilm regions to investigate region specific aspects of biofilm micro-rheology. Koza et al. (2009) studied the viscoelastic properties of $P$. fluorescens SBW25 biofilms cultivated at the airliquid interface by using a parallel plate rheometer. The stress amplitude sweep tests were conducted at $0.5 \mathrm{~Hz}$, yielding a shear modulus of $0.75 \mathrm{~Pa}$, which is close to what was observed in this present study. ${ }^{20}$ This proves that the non-destructive microrheological method described in this study is in good agreement with the classical yet destructive rheological methods (i.e., parallel plate rheometer) for the quantification of biofilm mechanical properties.

\section{Micro-rheological analysis of biofilm regions of interest}

Upon further examination of embedded beads within biofilm depths, beads were found to be localized within specific regions characterized as biofilm voids (zone I) and clusters (zone 2) (Fig. 5). Biofilm voids in this study were defined as areas with distinctively lower mCherry fluorescence and opaque in nature compared to surrounding bulk liquid areas (zone 3), while areas of high cell density characterized by the expression of mCherry fluorescent protein depicted as area 2 (Fig. 5a). Void and bulk liquid areas were differentiated by their distinct bead trajectories within these zones as illustrated in supplementary Fig. S3. 
$24 \mathrm{~h}$

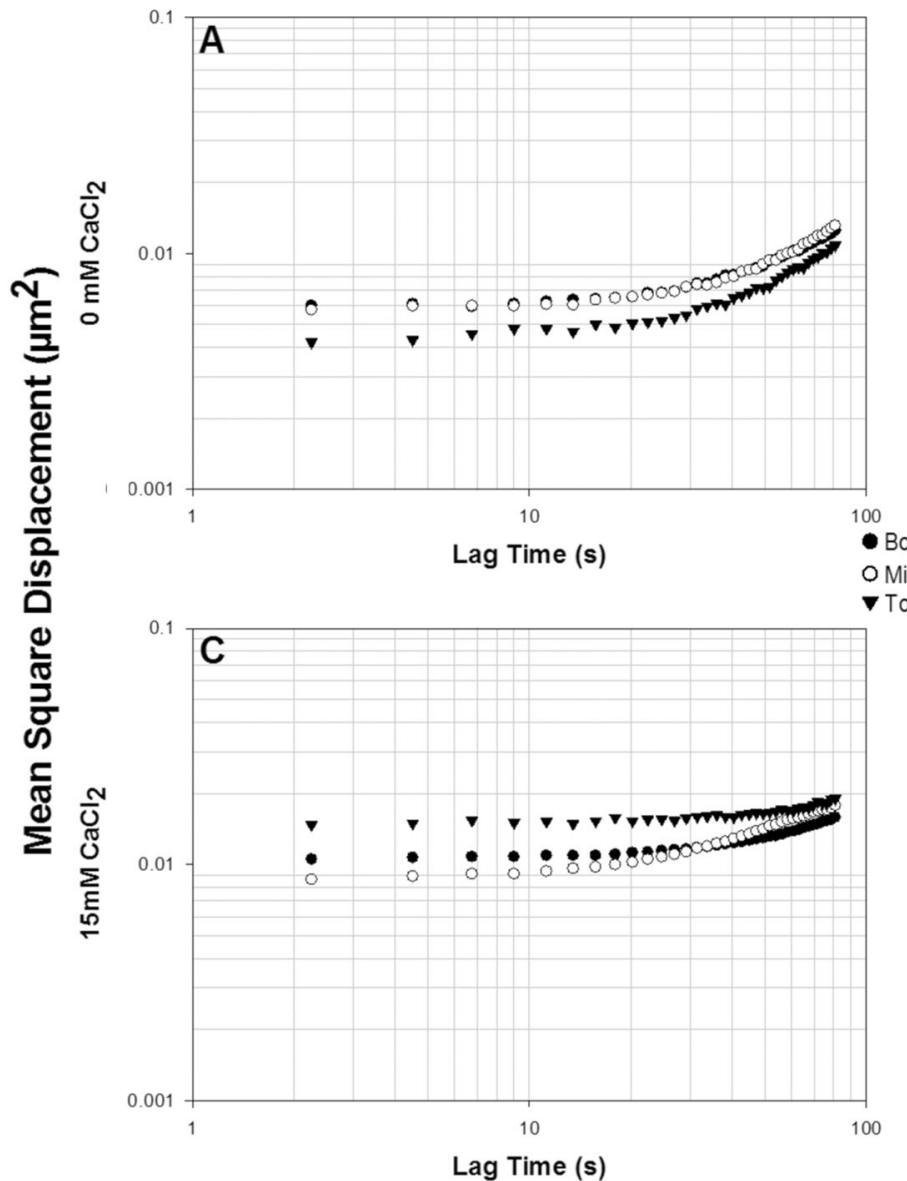

48h

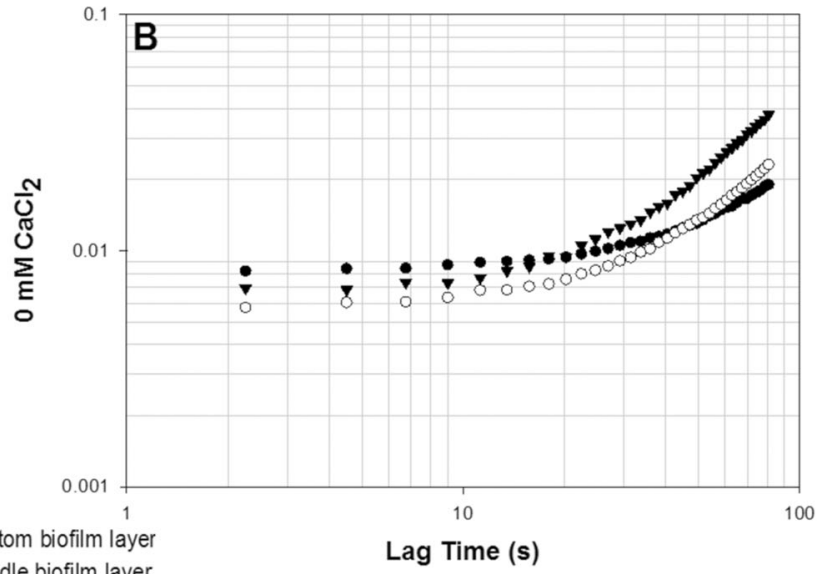

Lag Time (s)

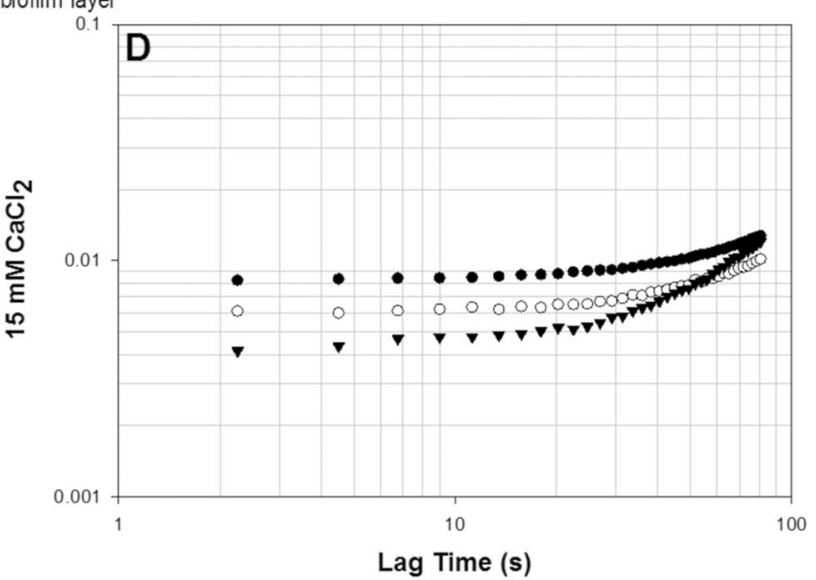

Fig. 3. MSD profiles of $1 \mu \mathrm{m}$ fluorescent beads within bottom, middle and top biofilm layers of $24 \mathrm{~h}$ (a, c) and $48 \mathrm{~h}$ (b, d) Pseudomonas fluorescens biofilms grown at $0 \mathrm{mM}(\mathbf{a}, \mathbf{b})$ and $15 \mathrm{mM}(\mathbf{c}, \mathbf{d}) \mathrm{CaCl}_{2}$ environments

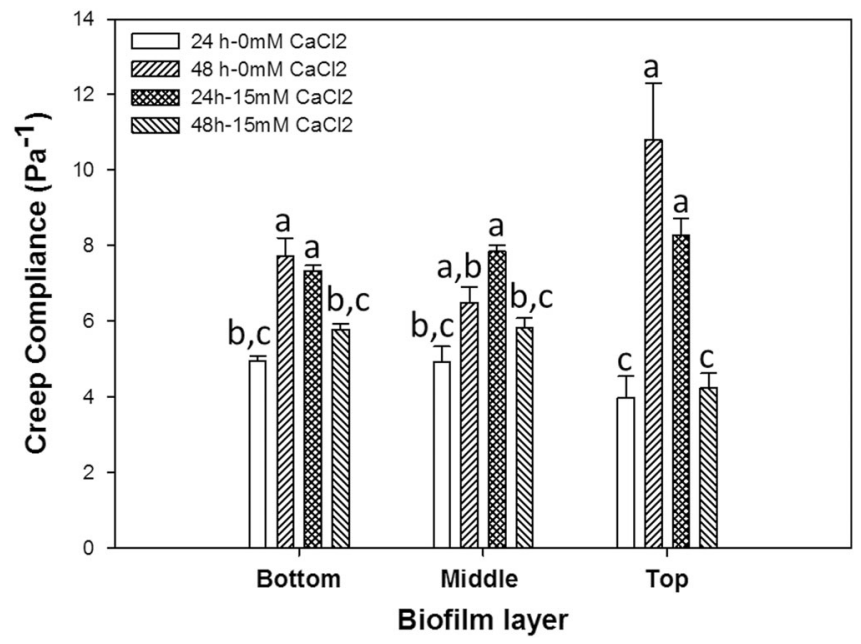

Fig. 4. Mean creep compliance of Pseudomonas fluorescens bottom, middle and top biofilm layers following $24 \mathrm{~h}$ and $48 \mathrm{~h}$-biofilm development under the absence and presence of $\mathrm{CaCl}_{2}$ environments. Means that do not share a letter are significantly different. Error bars represent standard error of the mean

The MSD analysis of fluorescent beads within biofilm layers was repeated, however this time by taking into consideration the spatial heterogeneities in each layer. By plotting the trajectories of each recorded bead within their respective field of view and biofilm layers, contour maps of corresponding creep compliance values were generated, delimitating zones of high and low ductility (Fig. 5b). These contour maps revealed that the rheological profile within the different layers were highly heterogeneous with areas of high ductility described by high creep compliance range (dark green) to stiffer zones of lower creep compliance values (light green). By overlapping the generated contour plots with corresponding CLSM micrographs (Fig. 5c) it was revealed that the void areas were characterized by high creep compliance values, while cluster areas were defined with lowest creep compliance. These results seem to suggest that the voids areas may be composed of a significant amount of exopolymeric substances compared to cluster areas. While void areas were distinctively composed of lower cell density (based on mCherry fluorescence), their high ductility can thus be attributed to low cell-to-EPS ratios. Cluster area having higher cell densities and therefore higher cell-to-EPS ratios was shown to be generally stiffer. These results add weight to the conclusions of a hypothesis described in a recent study where the cell-to-EPS ratios was found to influence the viscoelasticity of biofilms. ${ }^{4}$ While Safari et al. ${ }^{4}$ assessed the viscoelastic properties of $5 \%$ of the uppermost biofilm top layer, the force spectroscopy employed in that study was restricted by its limited (by depth) range of biofilm sampling. The microrheological method presented in this study goes beyond the limitations of force spectroscopy, and offers the potential for an improved understanding of region-specific material property characteristics at different biofilm layers. This was achieved by comparing the regional-based creep compliance values of the different biofilm layers as shown in Fig. 6 .

The region-based creep compliance analysis led to the rheological differentiation of clusters and void regions $(p<0.0001)$, where the former was found to be generally stiffer than void areas irrespective of biofilm layer or biofilm growth conditions and growth period (cf. Fig. S3 in the supplementary material section). Similar profiles were observed by Birjiniuk et al., in which embedded beads within biofilms were separated by their level of mobility and confinement. ${ }^{6}$ Interestingly the rheological dynamics 

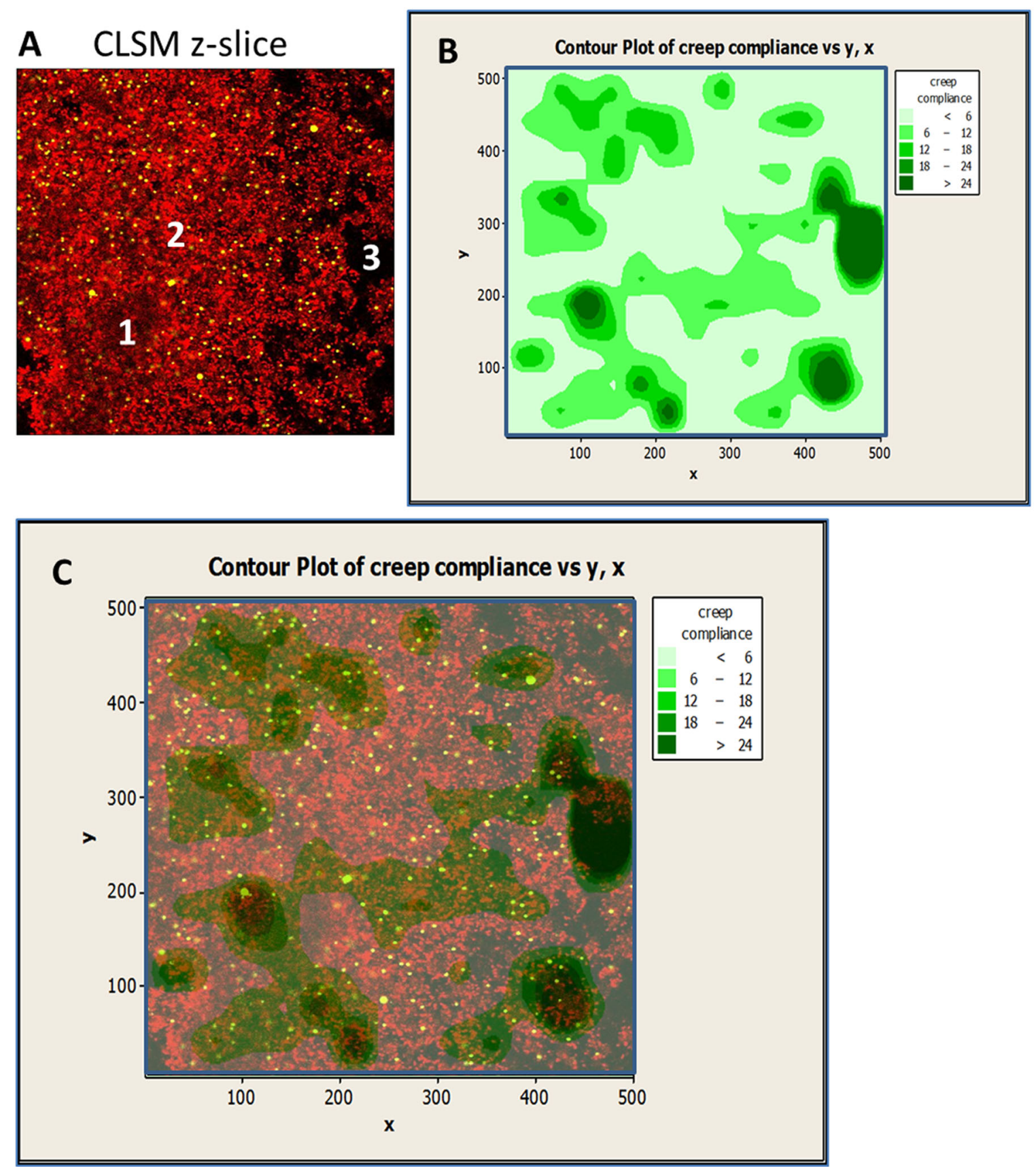

Fig. 5. Region-specific Creep compliance analysis. a Example of a horizontal plane (xy-) micrograph of a biofilm layer depicting mCherryexpressing $P$. fluoresence cells (red) and $1 \mu \mathrm{m}$ fluorescent beads (green). Zones 1, 2 identify biofilm areas of high low cell density and high cell density respectively. Zone 3 is recognized as surrounding bulk suspension. b Bead trajectories from zones 1 and 2 are used to calculate MSDs used to determine Creep compliance. Akima's polynomial method for the interpolation of creep compliance values for each fluorescent bead's xy-coordinate was used to generate contour maps. c By merging xy-micrographs and corresponding contour maps, region-specific creep compliance analysis is possible

profiles within voids and clusters showed similar trends to global rheological trends presented in Fig. 4. Moreover, results from regional creep compliance analysis also suggest that the creep compliance of biofilm void zones is the primary contributor to biofilm mechanical properties, contributing to the overall viscoelastic character. The low creep compliance profile of cluster areas may be linked to the higher density of cells there.

\section{CONCLUSIONS}

The use of $1 \mu \mathrm{m}$ beads for the purpose of microrheological measurements combined with confocal imaging of biofilm structure was successful. MSD measurements were used to determine biofilm creep compliances, which allowed visualisation of region-specific microrheological profiles within the biofilm. Compared to force spectroscopy or classical (bulk) rheological techniques, the micro-rheological method described in this study offers the possibility of investigating region-specific biofilm rheological properties in an in-situ and non-destructive manner. Future improvements to this technique could include localised chemical analysis of biofilms to determine whether the EPS within regions of distinct creep compliance profiles is related to either the type of EPS present in those regions or the degree of unrestricted displacement based on cell density. With the unmet need to control unwanted biofilms, understanding biofilm micro- 


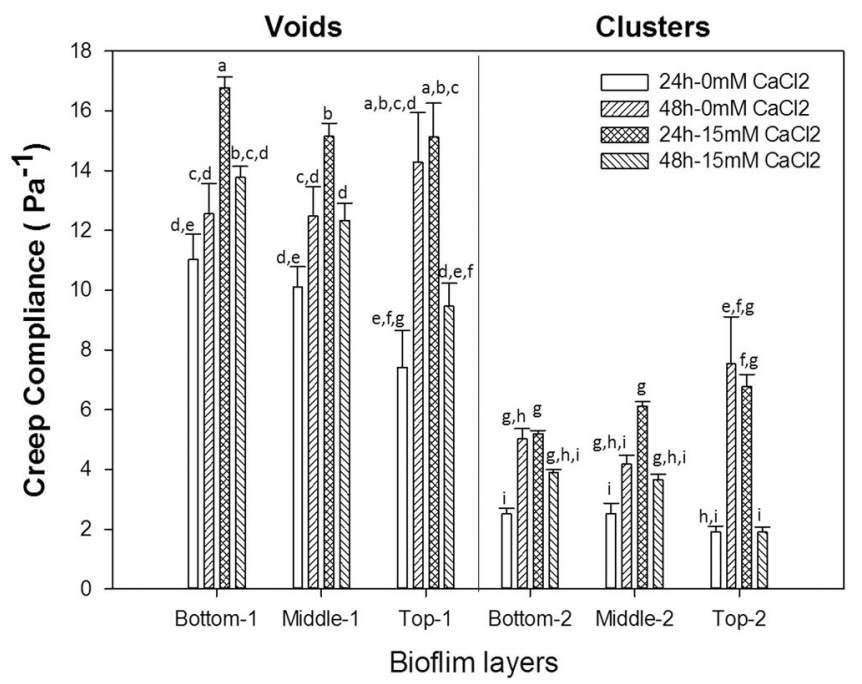

Fig. 6. Mean creep compliance of Pseudomonas fluorescens void and cell cluster regions within bottom, middle and top biofilm layers following $24 \mathrm{~h}$ and $48 \mathrm{~h}$-biofilm development under the absence and presence of $\mathrm{CaCl}_{2}$ environments. Means that do not share a letter are significantly different. Error bars represent standard error of the mean

rheological profiles is expected to become a crucial step in optimizing novel biofilm treatments and eradication strategies.

\section{ACKNOWLEDGEMENTS}

This research was supported by the European Research Council (ERC), project 278530, funded under the EU Framework Programme 7, as well as the Science Foundation Ireland under Grant Number: SFI 11/RFP.1/ENM/3145. The authors would like to thank Dr Ellen L Lagendijk from the Institute of Biology Leiden, Netherlands for the gift of the $P$. fluorescens PCL1701.

\section{COMPETING INTERESTS}

The authors declare that they have no competing interests.

\section{REFERENCES}

1. Stoodley, P., Cargo, R., Rupp, C., Wilson, S. \& Klapper, I. Biofilm material properties as related to shear-induced deformation and detachment phenomena. J. Ind. Microbiol. Biotechnol. 29, 361-367 (2002).

2. Wilking, J. N., Angelini, T. E., Seminara, A., Brenner, M. P. \& Weitz, D. A. Biofilms as complex fluids. MRS Bull. 36, 385-391 (2011).

3. Hall-Stoodley, L., Costerton, J. W. \& Stoodley, P. Bacterial biofilms: from the natural environment to infectious diseases. Nat. Rev. Microbiol. 2, 95-108 (2004).
4. Safari, A., Habimana, O., Allen, A. \& Casey, E. The significance of calcium ions on Pseudomonas fluorescens biofilms - a structural and mechanical study. Biofouling 30, 859-869 (2014).

5. Jones, W. L., Sutton, M. P., McKittrick, L. \& Stewart, P. S. Chemical and antimicrobial treatments change the viscoelastic properties of bacterial biofilms. Biofouling 27, 207-215 (2011).

6. Birjiniuk, A. et al. Single particle tracking reveals spatial and dynamic organization of the Escherichia coli biofilm matrix. New J. Phys. 16, 085014 (2014).

7. Chew, S. C. et al. Dynamic remodeling of microbial biofilms by functionally distinct exopolysaccharides. mBio 5, 1-11 (2014).

8. Galy, O. et al. Mapping of bacterial biofilm local mechanics by magnetic microparticle actuation. Biophys. J. 103, 1400-1408 (2012).

9. Rogers, S., Van Der Walle, C. \& Waigh, T. Microrheology of bacterial biofilms in vitro: Staphylococcus aureus and Pseudomonas aeruginosa. Langmuir 24, 13549-13555 (2008).

10. Stojković, B., Sretenovic, S., Dogsa, I., Poberaj, I. \& Stopar, D. Viscoelastic Properties of levan-DNA mixtures important in microbial biofilm formation as determined by micro-and macrorheology. Biophys. J. 108, 758-765 (2015).

11. Lagendijk, E. L., Validov, S., Lamers, G. E., De Weert, S. \& Bloemberg, G. V. Genetic tools for tagging Gram-negative bacteria with mCherry for visualization in vitro and in natural habitats, biofilm and pathogenicity studies. FEMS Microbiol. Lett. 305, 81-90 (2010).

12. King, E. O., Ward, M. K. \& Raney, D. E. Two simple media for the demonstration of pyocyanin and fluorescin. J. Lab. Clin. Med. 44, 301-307 (1954).

13. Beyenal, H., Lewandowski, Z. \& Harkin, G. Quantifying biofilm structure: Facts and fiction. Biofouling 20, 1-23 (2004).

14. Haralick, R. M. \& Shanmugam, K. Textural features for image classification. IEEE Trans. Syst. Man Cybern. 3, 610-621 (1973).

15. Shannon, C. E. A mathematical theory of communication. Bell Syst. Tech. J. 27, 623-656 (1948).

16. Vallotton, P. \& Olivier, S. Tri-track: Free software for large-scale particle tracking Microsc. Microanal. 19, 451-460 (2013).

17. Rich, J. P., McKinley, G. H. \& Doyle, P. S. Size dependence of microprobe dynamics during gelation of a discotic colloidal clay. J. Rheol. 55, 273-299 (2011).

18. Habimana, O., Meyrand, M., Meylheuc, T., Kulakauskas, S. \& Briandet, R. Genetic features of resident biofilms determine attachment of Listeria monocytogenes. Appl. Environ. Microbiol. 75, 7814-7821 (2009).

19. Stewart, P. S. \& Franklin, M. J. Physiological heterogeneity in biofilms. Nat. Rev. Microbiol. 6, 199-210 (2008).

20. Koza, A., Hallett, P. D., Moon, C. D. \& Spiers, A. J. Characterization of a novel air-liquid interface biofilm of Pseudomonas fluorescens SBW25. Microbiology 155, 1397-1406 (2009).

(c) (i) This work is licensed under a Creative Commons Attribution 4.0 International License. The images or other third party material in this article are included in the article's Creative Commons license, unless indicated otherwise in the credit line; if the material is not included under the Creative Commons license, users will need to obtain permission from the license holder to reproduce the material. To view a copy of this license, visit http://creativecommons.org/licenses/by/ $4.0 /$

(c) The Author(s) 2016

Supplementary Information accompanies the paper on the npj Biofilms and Microbiomes website (doi:10.1038/s41522-016-0005-y). 